\title{
BEM/OQM Formulation to Simulate Crack Problems ${ }^{1}$
}

C.A.R. VERA-TUDELA ${ }^{2}$, Departamento de Matemática, Universidade Federal Rural do Rio de Janeiro, UFRRJ, 23890-971 Seropédica, RJ, Brasil.

E.F. FONTES Jr., J, J.C.F. TELLES 4 , Programa de Engenharia Civil, Universidade Federal do Rio de Janeiro, COPPE, UFRJ, 21945-970 Rio de Janeiro, RJ, Brasil.

\begin{abstract}
Over the last few years the Boundary Element Method (BEM) has been successfully applied to linear elastic fracture mechanics problems (LEFM), involving static and dynamic cases. An approach to solve LEFM problems is presented in this work. The Numerical Green's Function is used at the fundamental solution together with the Operational Quadrature Method. Proceeding this way, there is no need to discretize the unloaded crack surface and the convolution integral is substituted by a Quadrature formula whose weights are computed using the Laplace transform of the fundamental solution and a linear multistep method. This solution strategy is here implemented to deal with problems associated with the scalar wave equation.
\end{abstract}

Keywords. Boundary element method, numerical Green's function, operational quadrature method.

\section{Introduction}

The initiation of cracks in structural elements and machines occurs frequently as a consequence of the production process coupled with small imperfections of the material. The study of cracks is important due to its direct effect in useful life predictions and overall resistance estimations of many structural parts.

The Boundary Element Method (BEM) [4] is an important tool in the study of problems governed by scalar wave equation and in problems related to elastodynamics. Several formulations have been developed and tested in the last few years with good practical results. The study of crack problems using a Numerical Green Function (NGF) [11] is an important alternative because it removes element interpolation from the crack surface. Such a formulation, coupled with the standard

\footnotetext{
${ }^{1}$ This work has been carried out with the support of the FAPERJ and the Fundo Setorial de Infra-Estrutura (CT-INFRA) with the $\mathrm{MCT} / \mathrm{CNPq}$ - Programa Primeiros Projetos and Apoio às Instituições de Ensino e Pesquisa Sediadas no Estado do Rio de Janeiro; the Universidade Federal Rural do Rio de Janeiro with "Grupos Emergentes" project.

2 candres@ufrrj.br.

${ }^{3}$ fontesjunior@coc.ufrj.br

${ }^{4}$ telles@coc.ufrj.br.
} 
BEM time-domain approach [12], is expected to present good accuracy and a good representation of the causality principle. To this end, the present work discusses first the NGF idea implemented to the equivalent scalar counterpart problem and leaves the extension to elastodynamic wave propagation as a future generalization. Hence, the BEM developed here addresses problems governed by the scalar wave equation and makes use of the fundamental solution in the Laplace domain to produce a direct solution to the associated problem in the time-domain. This approach is based on the Operational Quadrature Method (OQM) $[6,7,8]$.

\section{Scalar Wave Equation}

The scalar wave equation [10], is written as (in the absence of domain contributions):

$$
\nabla^{2} u(x, t)-\frac{1}{c^{2}} \frac{\partial^{2} u(x, t)}{\partial t^{2}}=0
$$

where $u(x, t)$ is the potential, $t$ is time and $c$ is the wave propagation velocity.

Boundary and initial conditions that define the particular solution to Eq. 2.1 are:

Boundary conditions $\left(x \in \Gamma ; \Gamma_{u} \cup \Gamma_{p}\right.$ is the boundary)

$$
\begin{aligned}
u(x, t) & =\bar{u}(x, t) & & x \in \Gamma_{u} \\
p(x, t) & =\frac{\partial u(x, t)}{\partial n}=\bar{p}(x, t) & & x \in \Gamma_{p}
\end{aligned}
$$

Initial conditions $(x \in \Omega ; \Omega$ is the domain)

$$
\begin{aligned}
& u(x, 0)=u_{0}(x) \\
& v(x, 0)=\left.\frac{\partial u(x, t)}{\partial t}\right|_{t=0}=v_{0}(x)
\end{aligned}
$$

In Eq. 2.2, $n$ is the coordinate in the boundary outward normal direction.

\section{The Operational Quadrature Method}

The Operational Quadrature Method (OQM) developed by Lubich [6, 7, 8] approximate the convolution of a function $y(t)$ in $u^{t}(t-\tau)$ and $f(t)$ by a discrete convolution, using only values of $\hat{u}^{t}(s)$ (Laplace transform of $u^{t}(t)$ ) and $f(t)$. This procedure is applied in the numerical resolution of the time domain boundary integral equation corresponding to the potential problem. It is defined as follows:

$$
y(t)=\int_{0}^{t} u^{t}(t-\tau) f(t) d \tau=u^{t}(t) * f(t)
$$

where $u^{t}(t)$ represents the fundamental solution in a time domain BEM. In this way, Eq. 3.1 can be written in a discretized form as: 


$$
y(n \Delta t)=\sum_{k=0}^{n} w_{n-k}(\Delta t) f(k \Delta t), \quad n=0,1, \ldots, N
$$

In Eq. 3.2, $w_{n}$ represents the integration weights determined by the coefficients of a complex variable power series in the Laplace transform $\hat{u}^{t}(s)$ of the fundamental solution $u^{t}(t)$ as follows:

$$
\hat{u}^{t}(s)=\hat{u}^{t}\left(\frac{\gamma(z)}{\Delta t}\right)=\sum_{n=0}^{\infty} w_{n}(\Delta t) z^{n}
$$

The coefficients of the power series are usually calculated with Cauchy's integral formula. After a polar coordinate transformation, this integral is approximated by a trapezoidal rule with $L$ equal steps $\frac{2 \pi}{L}$. This leads to:

$$
\begin{aligned}
w_{n}(\Delta t) & =\frac{1}{2 \pi i} \int_{|z|=\rho} \hat{u}^{t}\left(\frac{\gamma(z)}{\Delta t}\right) z^{-n-1} d z \\
& =\frac{\rho^{-n}}{L} \sum_{i=0}^{L-1} \hat{u}^{t}\left(\frac{\gamma\left(\rho e^{\frac{i l 2 \pi}{L}}\right)}{\Delta t}\right) e^{\frac{-i n l 2 \pi}{L}}
\end{aligned}
$$

where $\rho$ is the radius of a circle in the domain of analyticity of the function.

The function $\gamma(z)$, previously utilized in Eqs. 3.3 e 3.4, is the quotient of the polynomials generated by a linear multistep method. For instance, if such a method is employed to approximate a certain function $x(t)$, solution of the first order differential equation:

$$
\frac{d x(t)}{d t}=s x(t)+f(t) ; \quad x(0)=0
$$

Then, one has:

$$
x(t)=\sum_{j=0}^{k} \alpha_{j} x_{n-j}=\Delta t \sum_{j=0}^{k} \beta_{j}\left(s x_{n-j}+f((n-j) \Delta t)\right)
$$

Hence

$$
\gamma(z)=\frac{\alpha_{0}+\cdots+\alpha_{k} z^{k}}{\beta_{0}+\cdots+\beta_{k} z^{k}}
$$

The function $\gamma(z)$ characterizes the multistep method and must be $A(\alpha)$-stable with positive angle $\alpha$, stable in a neighborhood of infinity, strongly zero-stable, and consistent of order $p$. If an error $\delta$ is assumed in the computation of $\hat{u}^{t}(s)$ in Eq. 3.3 , the choice $\mathrm{L}=\mathrm{N}$ and $\rho^{N}=\sqrt{\delta}$ leads to an error in $w_{n}$ of order $O(\sqrt{\delta})$. 


\section{Boundary Integral Equation}

The Time-Domain boundary element equation corresponding to the scalar wave equation is written as:

$$
\begin{aligned}
4 \pi C(\xi) u(\xi, t)= & \int_{\Gamma} \int_{0}^{t^{+}} u^{t}(x, t ; \xi, \tau) p(x, \tau) d \tau d \Gamma(x) \\
& -\int_{\Gamma} \int_{0}^{t^{+}} p^{t}(x, t ; \xi, \tau) u(x, \tau) d \tau d \Gamma(x)
\end{aligned}
$$

where $u^{t}(x, t ; \xi, \tau)$ is the so-called fundamental solution and $p^{t}(x, t ; \xi, \tau)=\frac{\partial u^{t}}{\partial n}$. $C(\xi)$ is the usual free coefficient dependent on the location of $\xi$ (interior, $\Omega$ or boundary, $\Gamma$ ). Following Eq. 3.2, the convolution implicitly indicated in Eq. 3.7 is written as:

$$
\int_{\Gamma} \int_{0}^{t^{+}} u^{t}(x, t ; \xi, \tau) p(x, \tau) d \tau d \Gamma=\sum_{k=0}^{n} g_{n-k}^{j}(x, \xi, \Delta t) p_{k}^{j}(x), \quad n=0,1, \cdots, N
$$

and

$$
\int_{\Gamma} \int_{0}^{t^{+}} p^{t}(x, t ; \xi, \tau) u(x, \tau) d \tau d \Gamma=\sum_{k=0}^{n} h_{n-k}^{j}(x, \xi, \Delta t) u_{k}^{j}(x), \quad n=0,1, \cdots, N
$$

In which it was already assumed that the boundary is discretized in $J$ elements $\Gamma_{j}$. The weights $g_{n}$ and $h_{n}$ are computed by following a pattern similar to that presented in Eq. 3.4. For each element, Eqs. 4.2 and

4.3 are written as:

$$
g_{n}^{j}(x, \xi, \Delta t)=\frac{\rho^{-n}}{L} \sum_{l=0}^{L-1} \int_{\Gamma_{j}} \hat{u}^{t}\left(x, \xi, \frac{\gamma\left(\rho e^{\frac{i l 2 \pi}{L}}\right)}{\Delta t}\right) \phi^{j}(x) d \Gamma(x) e^{\frac{-i n l 2 \pi}{L}}
$$

and

$$
h_{n}^{j}(x, \xi, \Delta t)=\frac{\rho^{-n}}{L} \sum_{l=0}^{L-1} \int_{\Gamma_{j}} \hat{p}^{t}\left(x, \xi, \frac{\gamma\left(\rho e^{\frac{i l 2 \pi}{L}}\right)}{\Delta t}\right) \phi^{j}(x) d \Gamma(x) e^{\frac{-i n l 2 \pi}{L}}
$$

where $\phi^{j}(x)$ represents the interpolation functions defined in the boundary discretization. Finally, in the present work an error $\delta=10^{-10}$ was adopted and the function $\gamma(z)$ of order $p$, was taken as:

$$
\gamma(z)=\sum_{n=1}^{p} \frac{1}{n}(1-z)^{n}
$$




\section{The numerical Green's function}

The Green's function boundary integral equation, for crack problems, is obtained if the fundamental solution is written in terms of a superposition of the Kelvin solution plus a complementary part which provides satisfaction of the traction-free requirement over the crack surface. Figure 1 illustrates this idea and the traction-free condition removes the need to formally discretize the crack surface in the boundary element formulation [11].

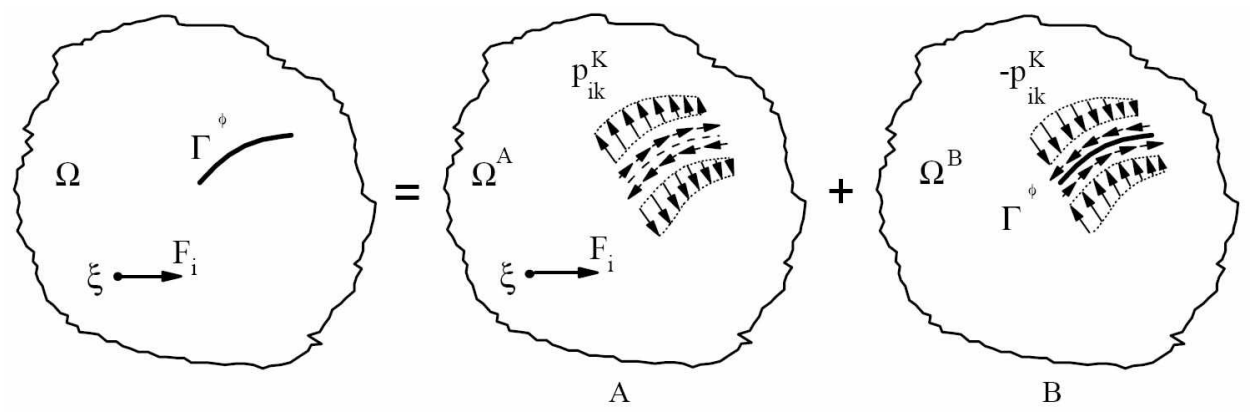

Figure 1: Unit point $P_{i}$ applied within infinite space with a traction-free crack. (A) Kelvin's problem; (B) Complementary problem.

The Green's function can be represented as

$$
\begin{aligned}
& u_{i j}^{G}(\xi, x)=u_{i j}^{K}(\xi, x)+u_{i j}^{c}(\xi, x) \\
& p_{i j}^{G}(\xi, x)=p_{i j}^{K}(\xi, x)+p_{i j}^{c}(\xi, x)
\end{aligned}
$$

where $u_{i j}^{G}(\xi, x)$ and $p_{i j}^{G}(\xi, x)$ are the fundamental displacement and tractions, in $j$ direction at the field point $x$ due to a unit point load applied at the source point $x$ in the $i$ direction. The superscripts $K$ and $c$ stand for Kelvin and complementary components, respectively, of the fundamental problem.

The Kelvin components $u_{i j}^{K}$ and $p_{i j}^{K}$ are known [3], the complementary displacements and tractions, $u_{i j}^{c}(\xi, x)$ and $p_{i j}^{c}(\xi, x)$, are the unknowns of problem (B) (see Figure 1). This solution can be written in terms of boundary integral equations as shown bellow [3]:

$$
\begin{aligned}
& u_{i j}^{c}(\xi, x)=\int_{\Gamma^{I}} p_{j k}^{K}(x, \zeta) c_{i k}(\xi, \zeta) d \Gamma(\zeta), \quad x \notin \Gamma^{I} \\
& p_{i j}^{c}(\xi, x)=\int_{\Gamma^{I}} P_{j k}^{K}(x, \zeta) c_{i k}(\xi, \zeta) d \Gamma(\zeta), \quad x \notin \Gamma^{I}
\end{aligned}
$$

where $c_{i k}(\xi, \zeta)=u_{i k}^{c}\left(\xi, \zeta^{S}\right)-u_{i k}^{c}\left(\xi, \zeta^{I}\right)$ is the crack opening displacements of the Green's function. Here, $S$ and $I$ stand for "superior" and "inferior" surfaces of the crack as indicated in Figure 2. The sign of the integrals depends on the chosen 


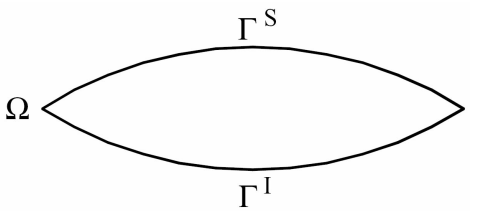

Figure 2: Infinite domain with crack boundary $\Gamma^{F}=\Gamma^{I} \cup \Gamma^{S}$.

surface of integration [11]; in this case $\Gamma^{I}$ has been adopted. The integral Eq. 5.3 is originated from the hypersingular or traction formulation.

Eqs. 5.2 and 5.3 produce the complementary displacements and tractions as a function of the fundamental crack openings. In order to solve the problem, it is therefore necessary to determine the crack opening displacements.

Figure 1 shows that the natural conditions of problem (B) are prescribed, hence the limit of Eq. 5.3 as $x \rightarrow \Gamma^{I}$ can be obtained to produce a hyper-singular boundary integral equation for the problem. This procedure has been discussed before [11, 12] and yields in the limit:

$$
p_{i j}^{c}(\xi, \bar{\zeta})=F p \int_{\Gamma^{I}} P_{j k}^{K}(\bar{\zeta}, \zeta) c_{i k}(\xi, \zeta) d \Gamma(\zeta), \quad \bar{\zeta} \in \Gamma^{I}
$$

where the symbol $F p \int$ indicates Hadamard's finite part integral.

Since, $p_{i j}^{c}(\xi, \bar{\zeta})=-p_{i j}^{K}(\xi, \bar{\zeta})$ Eq. 5.4 produces a hypersingular boundary integral equation for the unknown crack opening displacements:

$$
F p \int_{\Gamma^{I}} P_{j k}^{K}(\bar{\zeta}, \zeta) c_{i k}(\xi, \zeta) d \Gamma(\zeta)=-p_{i j}^{K}(\xi, \bar{\zeta})
$$

Eq. 5.5 can now be solved by a standard weighted residual method, using the point collocation technique. Therefore, using the Dirac delta function to weight the equation at a certain number of points $\bar{\zeta}_{m}(m=1, \cdots, M)$ on $\Gamma^{I}$, the following equations are found:

$$
F p \int_{\Gamma^{I}} P_{j k}^{K}\left(\bar{\zeta}_{m}, \zeta\right) c_{i k}(\xi, \zeta) d \Gamma(\zeta)=-p_{i j}^{K}\left(\xi, \bar{\zeta}_{m}\right), \quad m=1, \cdots, M
$$

Standard Gauss quadrature, in principle, cannot calculate finite part integrals. Hence, a rectifying scheme can be adopted in the following fashion [12]:

$$
\sum_{n=1}^{N}|J|_{n} P_{j k}^{K}\left(\bar{\zeta}_{m}, \zeta_{n}\right) c_{i k}\left(\xi, \zeta_{n}\right) W_{n}-E_{i j}\left(\xi, \bar{\zeta}_{m}\right)=-p_{i j}^{K}\left(\xi, \bar{\zeta}_{m}\right), \quad m=1, \cdots, M
$$

where $|J|_{n}$ is the Jacobian of the transformation to the standard quadrature interval and $\zeta_{n}$ the corresponding point at the Gauss station $n, W_{n}$ is the associated weighting factor at this station and $N$ is the total number of integration points. The correcting term, associated to the singular behaviour of the integrand, is $E_{i j}\left(\xi, \bar{\zeta}_{m}\right)$. 
In order to define $E_{i j}$, the crack opening displacements $c_{i k}$ can be expanded in Taylor's series about the point $\bar{\zeta}_{m}$ :

$$
\begin{aligned}
c_{i k}(\xi, \zeta)= & c_{i k}\left(\xi, \bar{\zeta}_{m}\right)+\frac{\partial c_{i k}\left(\xi, \bar{\zeta}_{m}\right)}{\partial \Gamma(\zeta)}\left[\Gamma(\zeta)-\Gamma\left(\bar{\zeta}_{m}\right)\right] \\
& +\frac{1}{2} \frac{\partial^{2} c_{i k}\left(\xi, \bar{\zeta}_{m}\right)}{\partial \Gamma(\zeta)^{2}}\left[\Gamma(\zeta)-\Gamma\left(\bar{\zeta}_{m}\right)\right]^{2}+\cdots
\end{aligned}
$$

Then, the next step is to substitute Eq. 5.8 in Eq. 5.6. Since $P_{j k}^{K}=O\left(r^{-2}\right)$ for 2-D applications, only the first two terms of the series produce singular behaviour; the first generates a finite part integral and the second a Cauchy principal value. Hence, $E_{i j}$ can be defined as

$$
E_{i j}\left(\xi, \bar{\zeta}_{m}\right)=c_{i k}\left(\xi, \bar{\zeta}_{m}\right) e_{j k}^{(1)}+\frac{\partial c_{i k}\left(\xi, \bar{\zeta}_{m}\right)}{\partial \Gamma(\zeta)} e_{j k}^{(2)}
$$

where

$$
e_{j k}^{(1)}=\sum_{n=1}^{N}|J|_{n} P_{j k}^{K}\left(\bar{\zeta}_{m}, \zeta_{n}\right) W_{n}-F p \int_{\Gamma^{I}} P_{j k}^{K}\left(\bar{\zeta}_{m}, \zeta\right) d \Gamma(\zeta)
$$

and

$$
\begin{aligned}
e_{j k}^{(2)}= & \sum_{n=1}^{N}|J|_{n} P_{j k}^{K}\left(\bar{\zeta}_{m}, \zeta_{n}\right)\left[\Gamma\left(\zeta_{n}\right)-\Gamma\left(\bar{\zeta}_{m}\right)\right] W_{n} \\
& -C p \int_{\Gamma^{I}} P_{j k}^{K}\left(\bar{\zeta}_{m}, \zeta\right)\left[\Gamma(\zeta)-\Gamma\left(\bar{\zeta}_{m}\right)\right] d \Gamma(\zeta)
\end{aligned}
$$

The finite part and Cauchy principal value $\left(C p \int\right)$ indicated as the last term of Eqs 5.10 and 5.11 are to be computed either analytically or using accurate numerical schemes.

In order to generate a square system of equations for $c_{i k}$ at the Gauss point positions, the number of collocation points is taken to be the same as the number of Gauss integration ones $(M=N)$, leading to

$$
\sum_{n=1}^{N}|J|_{n} P_{j k}^{K}\left(\bar{\zeta}_{m}, \zeta_{n}\right) c_{i k}\left(\xi, \zeta_{n}\right) W_{n}-E_{i j}\left(\xi, \bar{\zeta}_{m}\right)=-p_{i j}^{K}\left(\xi, \bar{\zeta}_{m}\right), \quad m=1, \cdots, N
$$

Careful examination of index expansions in Eq. 5.12 indicates that the system of $4 N$ equations can be subdivided further into two systems, of dimensions $2 N$, sharing the same square matrix $\mathbf{S}$, as is showed in the next equation:

$$
\mathbf{S c}_{i j}(\xi)=\mathbf{p}_{i j}(\xi)
$$

It is important to note that matrix $\mathbf{S}$ is only a function of crack geometry and remains the same for any position and direction of the unit point load. Furthermore, Eq. 5.13 is repeatedly solved applying just back substitution in a Gauss solution routine to calculate all needed $\mathbf{c}_{i j}(\xi)$ vectors. 
Finally, the numerical Green's function, for a crack simulation, is written using Eqs 5.2 and 5.3 and the calculated fundamental crack opening displacements at the discrete standard Gauss point positions:

$$
\begin{aligned}
& u_{i j}^{G}(\xi, x)=u_{i j}^{K}(\xi, x)+\sum_{n=1}^{N}|J|_{n} p_{j k}^{K}\left(x, \zeta_{n}\right) c_{i k}\left(\xi, \zeta_{n}\right) W_{n} \\
& p_{i j}^{G}(\xi, x)=p_{i j}^{K}(\xi, x)+\sum_{n=1}^{N}|J|_{n} P_{j k}^{K}\left(x, \zeta_{n}\right) c_{i k}\left(\xi, \zeta_{n}\right) W_{n}
\end{aligned}
$$

\section{Numerical Examples}

\subsection{Simulation of linear discontinuity in an infinite medium}

This example consists of a linear discontinuity in an infinite medium with a unit time step load. The problem is here studied in dimensionless form, adopting a total discontinuity length of $2 a=4$ and the time was divided in three different values of $\Delta t$ represented by the $\beta$ parameter $\left(\beta=c \frac{\Delta t}{l} ; c=1\right.$ is the wave velocity and $l$ is the smaller element length). The total time was 12 . The results are compared with a time-domain boundary element formulation [9] using 120 linear elements to represent half of the symmetric problem. For the Green's function results 52 quadratic elements, to represent the complete problem (symmetry was not taken into account), have been used. Figure 3 shows the solutions for the three different values of $\beta$.

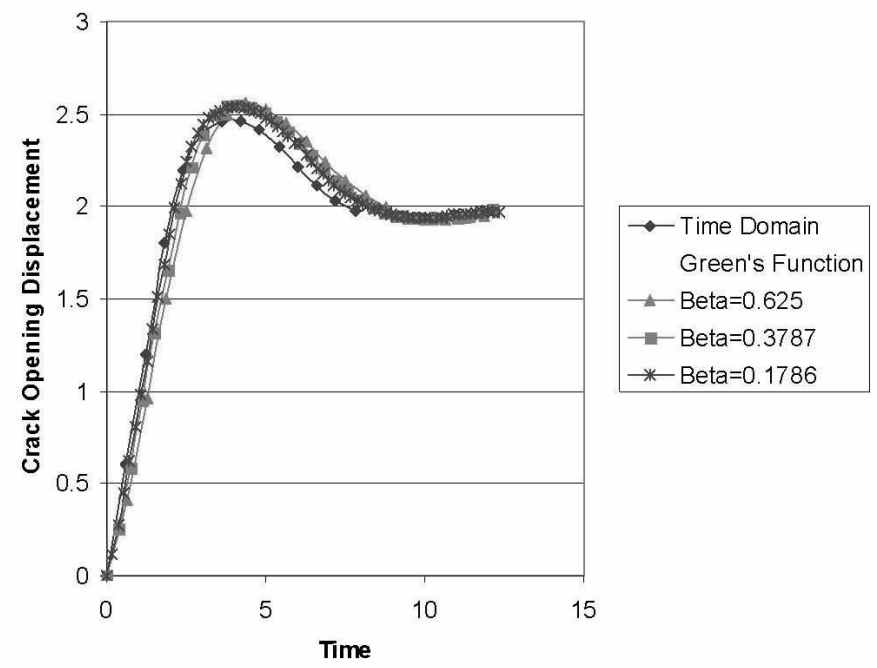

Figure 3: Simulation of linear discontinuity for infinite medium and different values of $\beta$. 


\subsection{Rectangular plate with a central discontinuity}

In this dimensionless example, the rectangular plate with a central discontinuity shown in Figure 4 is loaded along the smaller opposite boundaries at time $t=$ 0 by a constant load exhibiting Heaviside time dependent variation. The total discontinuity length is $2 a=4$; the dimensions of the rectangular plate are $2 h=16$, $2 b=10$. The discretization adopted was 52 quadratic elements with double nodes on the corners and $c=1$.

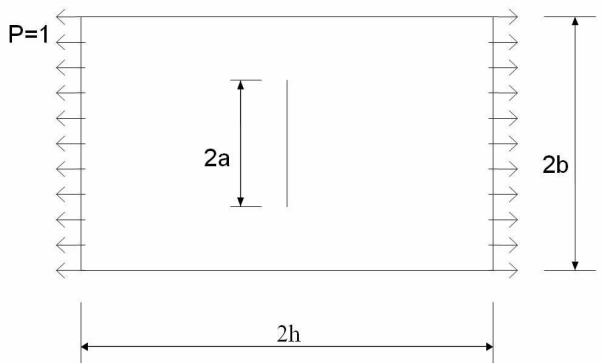

Figure 4: Geometry of the rectangular plate with a central discontinuity.

Initially, only the solutions for the NGF-OQM BEM formulation using different values of $\beta$ are compared with the standard (half the problem discretized) OQMBEM [10] approach. The results are shown in Figure 5.

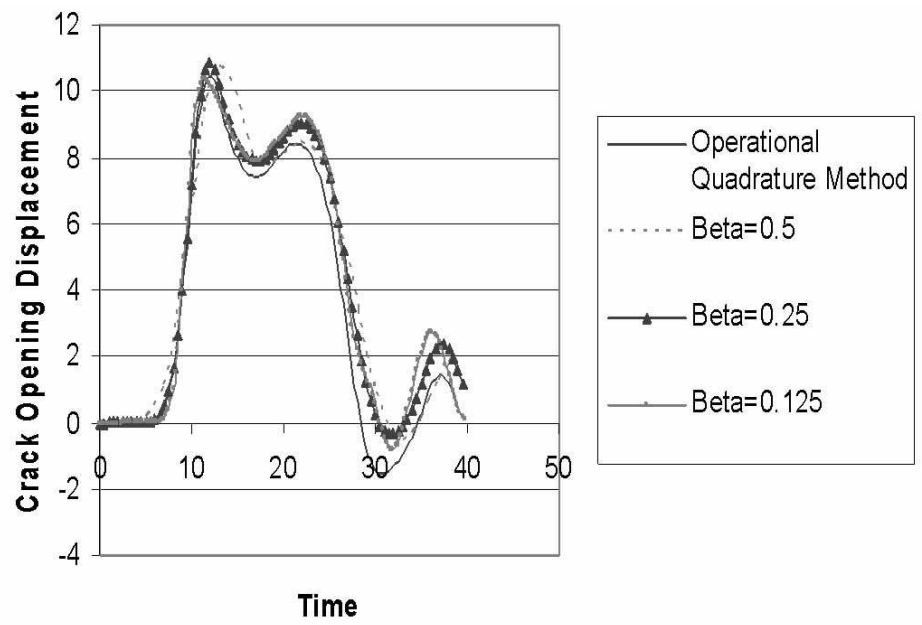

Figure 5: Results for different values of $\beta$.

In the second comparison the solution for the TD-BEM formulation has been added. Like the OQM-BEM, implicit symmetry (i.e., half the problem is discretized) is necessary to represent the discontinuity. In fact, for the TD-BEM, the boundary 
is discretized with 120 linear elements of which 8 are used to represent the discontinuity; for the OQM-BEM, 52 linear elements are defined with 10 to represent the discontinuity. The $\beta$ parameter was equal to 0.5 and the total time 35 . Figure 6 depicts the results of this second comparison.

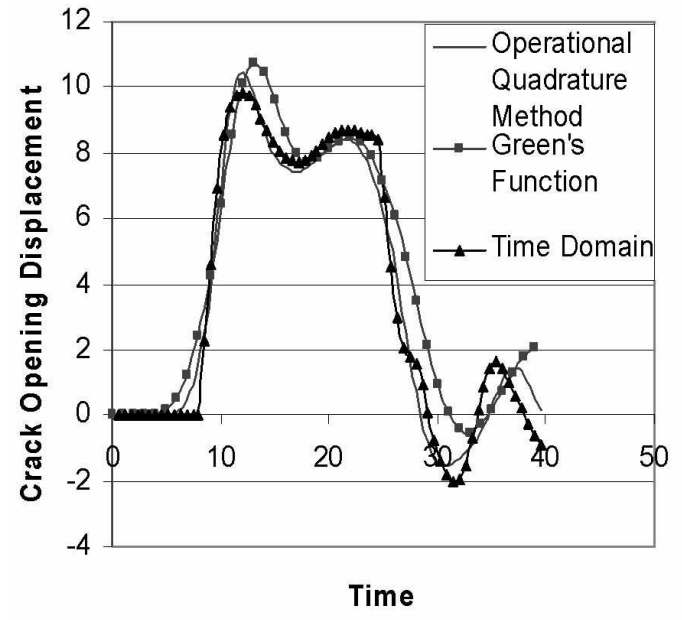

Figure 6: Plate with a central discontinuity: COD for three formulations.

\section{Conclusion}

The Numerical Green's Function developed in this work, together with the Operational Quadrature Method, has been described to potential problems. A series of examples illustrates the accuracy obtained when compared to Time-Domain Boundary Element formulation and the standard OQM-BEM, even for values of the dimensionless time step $\beta$ outside the usually recommended [9] range of $0.3-0.6$. The results confirm this recommendation but further studies must be performed to analyze the effect of the boundary discretization and the $\beta$ parameter.

Resumo. Nos últimos anos, o Método dos Elementos de Contorno (MEC) tem sido aplicado com êxito em problemas da Mecânica da Fratura Linear Elástica (MFLE), em aplicações estáticas, assim como dinâmicas. Uma aproximação para resolver problemas da MFLE é apresentada neste trabalho. A Função de Green numérica é usada como uma solução fundamental junto com o Método da Quadratura na Convolução. Desta forma, não existe a necessidade de discretizar a superfície da trinca descarregada e a integral de convolução é substituída por uma fórmula de Quadratura cujos pesos são computados usando a transformada de Laplace da solução fundamental e uma estratégia de avanço no tempo. A método de solução, aqui implementado, está direcionado para resolver problemas com a equação escalar da onda.

Palavras-Chave. Método dos elementos de contorno, função de Green numérica, método da quadratura na convolução. 


\section{References}

[1] A.I. Abreu, J.A.M. Carrer, W.J. Mansur, Scalar wave propagation in 2-D: a BEM formulation based on the operational quadrature method, Engineering Analysis with Boundary Elements, 27, No. 2 (2003), 101-105.

[2] L.P.S. Barra, J.C.F. Telles, A hyper-singular numerical Green's function generation for BEM applied to dynamic SIF problems, Engineering Analysis with Boundary Elements, 23, (1999), 77-87.

[3] G.E. Blandford, A.R. Ingraffea, J.A. Ligget. Two-dimensional stress intensity factor computation using the boundary element method, Int. J. Numer. Methods Eng., 17 (1981), 387-404.

[4] C.A. Brebbia, J.C.F. Telles, L.C. Wrobel, "Boundary Elements Techniques: Theory and Applications", Springer-Verlag, Berlin, 1984.

[5] S. Guimarães, J.C.F. Telles, On the hyper-singular boundary element formulation for fracture mechanics applications, Engineering Analysis with Boundary Elements, 13, (1994), 353-363.

[6] C. Lubich, Convolution quadrature and discretized operational calculus II, $\mathrm{Nu}$ mer. Math., 52 (1988), 413-425.

[7] C. Lubich, Convolution quadrature and discretized operational calculus I, $\mathrm{Nu}$ mer. Math. , 52 (1988), 129-145.

[8] C. Lubich, On the multistep time discretization of linear initial-boundary value problems and their boundary integral equation, Numer. Math., 67 (1994), 365389 .

[9] W.J. Mansur, "A Time-stepping Technique to Solve Wave Propagation Problems Using the Boundary Element Method", Ph.D. Thesis, University of Southampton, England, 1983.

[10] P.M. Morse, H. Feshbach, "Methods of Theoretical Physics", McGraw-Hill, New York, 1953.

[11] J.C.F. Telles, G.S. Castor, S. Guimarães, A numerical Green's function approach for boundary elements applied to fracture mechanics, International Journal for Numerical Methods in Engineering, 38 (1995), 3259-3274.

[12] J.C.F. Telles, S. Guimarães, Green's function: a numerical generation for fracture mechanics problems via boundary elements, Comput. Methods Appl. Mech. Eng., 188 (2000), 847-858. 